\title{
An Exact Algorithm for the Servers Allocation, Capacity and Flow Assignment Problem with Cost Criterion and Delay Constraint in Wide Area Networks
}

\author{
Marcin Markowski and Andrzej Kasprzak \\ Wroclaw University of Technology, Chair of Systems and Computer Networks \\ Wybrzeze Wyspianskiego 27, 50-370 Wroclaw, Poland \\ \{marcin.markowski, andrzej.kasprzak\} @pwr.wroc.pl
}

\begin{abstract}
The paper deals with the problem of simultaneously assignment of server's allocation, channel capacities and flow routes in the wide area network in order to minimize the criterion composed of the leasing capacity cost and the building cost of the network. An exact algorithm, based on the branch and bound method, is proposed to solve the problem. Some interesting features, observed during the computational experiments are reported.
\end{abstract}

Keywords: Server allocation, CFA problem, Wide Area Networks.

\section{Introduction}

The optimization problems, considered always when the wide area network must be built or modernized, consist in assignment of resource allocation (i.e. servers, replicas of servers), capacities of channels and flow routes. The optimal arrangement of resources and optimal choice of capacities and flow routes let us obtain the most efficient and economical solution. Designing of the wide area computer networks is always a compromise between the quality of service in the network [1] and the costs needed to build and to support the network. Then those two criterions are often used during the designing process, usually one of them is the optimizing criterion and the other is considered as constraint. In our previous papers [2, 3, 4] we have considered two-criteria and three-criteria problems based on designing the wide area networks, assuming that the maximal support cost of the network is bounded. Sometimes it is useful to formulate the optimizing problem in the other way - how to minimize the cost of the network, when the acceptable quality level is known. Then in the paper the problem of server's allocation, capacity and flow assignment with the cost criterion and delay constraint is considered. In our opinion it is well-founded to consider two kind of cost: the building cost of the network, borne once and the supporting cost, borne regularly. Then the criterion function is composed of two ingredients: the regular channel capacity leasing cost and the disposable server cost. We assume that maximal acceptable total average delay in the network is given as the constraint. Then the problem considered here may be formulated as follows: 
given: user allocation at nodes, set of possible nodes for each server, maximal value of the total average delay in the network, traffic requirements useruser and user-server, set of capacities and their costs for each channel,

minimize: linear combination of the capacity leasing cost and server cost, over: $\quad$ servers allocation, channel's capacity and multicommodity flow,

subject to: multicommodity flow constraints, channel capacities constraints, server allocation constraints, total average delay constraint.

We consider the discrete cost-capacity function because it is the most important from practical point of view for the reason that the channels capacities can be chosen from the sequence defined by ITU-T recommendations. Such formulated problem is NP-complete as more general than the capacity and flow assignment problem (CFA) with discrete cost-capacity function which is NP-complete [5].

The literature focusing on the server's allocation, capacity and flow assignment problem is very limited. Some algorithms for this problem with different delay criterion may be found in $[2,3,4,6]$. The formulated here problem uses cost criterion and take into account the maximal acceptable average delay in Wide Area Network as constraint. In the literature such formulated problem has not been considered yet.

\section{Problem Formulation}

Let $X_{r}$ be the set of binary variables determining capacities of channels and $Y_{r}$ be the set of binary variables determining servers allocation at nodes [3, 4]. $\left(X_{r}, Y_{r}\right)$ is called a selection. Let $\Re$ be the family of all selections satisfying assumed constraint. Let $U\left(Y_{r}\right)$ be the server cost (the connecting cost of all servers at nodes) and $T\left(X_{r}, Y_{r}\right)$ be the minimal average delay per packet in WAN given by the Kleinrock's formula [7]. Let $T \max$ be the maximal acceptable average delay per packet. Let $Q\left(X_{r}, Y_{r}\right)=\alpha D\left(X_{r}\right)+\beta U\left(Y_{r}\right)$, where $\alpha$ and $\beta$ are positive coefficients $\alpha, \beta \in[0,1]$, $\alpha+\beta=1$. Then, the considered problem is formulated as follows:

subject to

$$
\min _{\left(X_{r}, Y_{r}\right)} Q\left(X_{r}, Y_{r}\right)
$$

$$
\begin{gathered}
\left(X_{r}, Y_{r}\right) \in \Re \\
T\left(X_{r}, Y_{r}\right) \leq T^{\max }
\end{gathered}
$$

\section{The Branch and Bound Algorithm}

The problem (1-3) is NP-complete. Then the branch and bound method can be used to construct the exact algorithm for solving the considered problem. The detailed description of the calculation scheme of the branch and bound method may be found in the paper [8]. The branch and bound method involves constructing two important operations specific for the problem (1-3): lower bound and branching rules.

The lower bound of the criterion function for every possible successor $\left(X_{s}, Y_{s}\right)$ generated from $\left(X_{r}, Y_{r}\right)$ must be computed. Since traffic requirements in the network 
depend on server allocation, then obtaining the lower bound for the problem (1-3) is difficult. We propose, the lower bound may be obtained by relaxing some constraints and by approximating the discrete cost-capacity curves with the lower linear envelope [5].

The purposes of the branching rules is to find the variable from $\left(X_{r}, Y_{r}\right)$ for complementing and generating a successor of the selection $\left(X_{r}, Y_{r}\right)$ with the least possible value of criterion function $Q$. The choice criterions should be constructed in such a way that complementing reduces value of criterion $Q$ and the increase of total average delay in the network is as minimal as possible $[3,4]$.

\section{Computational Results}

The presented algorithm was implemented in $\mathrm{C}++$ code and extensive numerical experiments, for many different networks, have been performed. Experiments were conducted with two main purpose in mind: first, to examine the impact of various problem parameters on the solution (i.e. on the value of criterion $Q$ ) and second, to test the computational efficiency of the algorithm.

The typical dependence of the optimal value of $Q$ on $T^{\max }$ for different values of parameters $\alpha$ and $\beta$ is presented in the Fig. 1. It follows from computational experiments that the dependence of $Q$ on $T^{\max }$ is decreasing function. The following conclusion follows from the computer experiments (Fig.1).

Conclusion 1. There exists such acceptable total average delay $T_{*}^{\max }$, that problem (1-3) has the same solution for each $T^{\max }$ greater or equal to $T_{*}^{\max }$.

Let $N T=\left(\left(T_{\max }-T_{\min }\right) /\left(T_{*}^{\max }-T_{\min }\right)\right) \cdot 100 \%$ be the normalized maximal acceptable total average delay per packet in the network - problem (1-3) has no solution for $T^{\max }<T_{\min }$. Normalized value let us compare results obtained for different network topologies. Let $P(u, v)$, in percentage, be the arithmetic mean of the relative number of iterations for $N T \in[\mathrm{u}, \mathrm{v}]$ calculated for all considered networks. Fig. 2 shows the dependency of $P$ on divisions $[0 \%, 10 \%), \ldots$, [90\%,100\%] of NT. It follows from Fig. 2 that the exact algorithm is especially effective from computational point of view for $N T \in[50 \%, 100 \%]$.

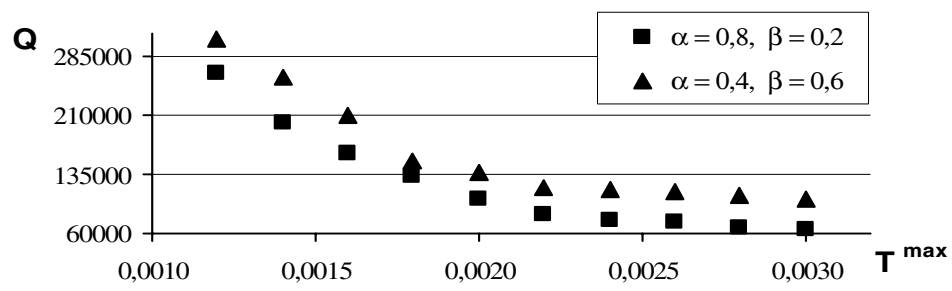

Fig. 1. Typical dependence of the optimal value of $Q$ on $T^{\max }$ 


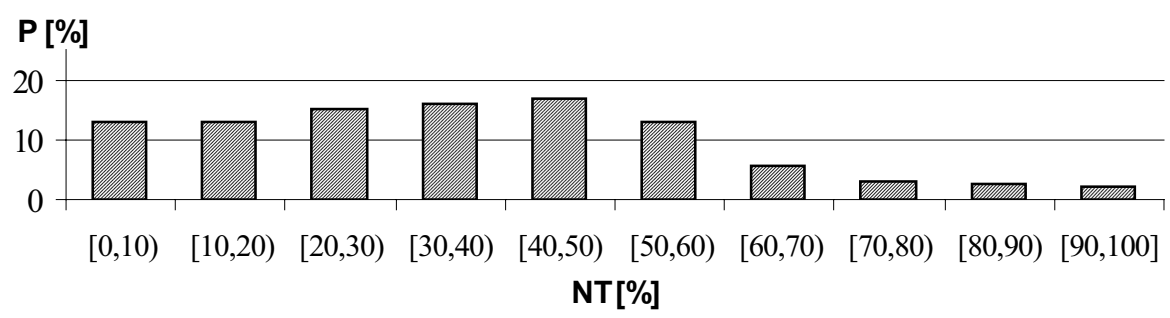

Fig. 2. The dependence of $P$ on normalized maximal average delay per packet $N T$

\section{Conclusion}

In the paper the exact algorithm for solving the server allocation, channel capacities and flow assignments problem with cost criterion and delay constraint is proposed. Such formulated problem has not been considered in the literature yet. It follows from computational experiments that the presented algorithm is effective from computational point of view for greater values of acceptable average delay in the network (Fig. 2). Moreover, we are of the opinion that the Wide Area Network property formulated as conclusion 1 is very important from practical point of view. This property shows that the values of the acceptable average delay are limited.

This work was supported by a research project of The Polish State Committee for Scientific Research in 2005-2007.

\section{References}

1. Walkowiak, K.: QoS Dynamic Routing in Content Delivery Network, Lecture Notes in Computer Science, Vol. 3462 (2005), 1120-1132

2. Markowski, M., Kasprzak, A.: An Exact Algorithm for Host Allocation, Capacity and Flow Assignment Problem in WAN, Internet Technologies, Applications and Societal Impact, Kluwer Academic Publishers, Boston (2002), 73-82

3. Markowski, M., Kasprzak, A.: The Web Replica Allocation and Topology Assignment Problem in Wide Area Networks: Algorithms and Computational Results, Lecture Notes in Computer Science, Vol. 3483 (2005), 772-781

4. Markowski, M., Kasprzak, A., The Three-Criteria Servers Replication and Topology Assignment Problem in Wide Area Networks, Lecture Notes in Computer Science, Vol. 3982 (2006), 1119-1128

5. Pioro, M., Medhi, D.: Routing, Flow, and Capacity Design in Communication and Computer Networks, Elsevier, Morgan Kaufmann Publishers, San Francisco (2004)

6. Chari, K.: Resource Allocation and Capacity Assignment in Distributed Systems, Computers Ops Res., Vol. 23, No. 11 (1996), 1025-1041

7. Fratta, L., Gerla, M., Kleinrock, L.: The Flow Deviation Method: an Approach to Store-andForward Communication Network Design, Networks 3 (1973), 97-133

8. Wolsey, L.A.: Integer Programming. Wiley-Interscience, New York (1998) 